\title{
THE USE OF THE “GAME RULES" GENRE IN THE ENGLISH TEACHING FOR CHILDREN
}

\author{
Ana Beatriz Maehashi Ferreira (Letras Inglês - UEL) ana maehashi@hotmail.com \\ Juliana Reichert Assunção Tonelli (UEL/PPGEL/MEPLEM) \\ teacherjuliana@uol.com.br
}

Esse trabalho é fruto de nossa prática docente no Centro Estadual de Educação Infantil pertencente à Universidade Estadual de Londrina. A pesquisa foi desenvolvida com alunos de 5 anos que tinham aulas de inglês com alunos-professores da mesma Universidade. O objetivo geral do estudo foi investigar a importância do domínio do gênero textual "regras de jogo" - no nosso caso, o jogo de tabuleiro - para a realização das atividades. Esta pesquisa, de natureza qualitativa, baseia-se na metodologia de pesquisa-ação (SAGOR, 2000; LEWIN, 1948). Os dados utilizados foram os diários reflexivos escritos pelos alunos-professores no decorrer do estágio obrigatório, locus principal de nossa investigação. Os resultados mostraram que o conhecimento do gênero textual possibilitou o engajamento efetivo dos alunos no evento discursivo proposto, atingindo assim o objetivo principal da atividade: revisar conteúdos referentes ao semestre anterior. Além disso, percebemos que, o fato de os alunos terem sido expostos ao gênero em questão possibilitou uma aprendizagem mais significativa da língua inglesa.

Palavras-chave: crianças, gêneros textuais, jogo de tabuleiro.

\section{INTRODUÇÃO}

O campo do ensino de inglês como língua estrangeira ${ }^{1}$ para crianças vem sendo investigado por diversos autores, Cristovão e Gamero (2009), Chaguri (2009), Figueira (2010), Chaguri e Tonelli (2014), por exemplo, postulam que uma maneira produtiva de ensinar a Língua Inglesa (doravante LI) para crianças é o uso de jogos e atividades lúdicas.

Tonelli (2013, p 312) postula que “(...) é por meio de aprendizagem significativa e da cooperação e participação 'do outro' que as crianças aprendem uma LE”, por essa razão, em nosso contexto de estágio, tivemos a intenção de fornecer aos alunos ambientes de sala de aula nos quais eles seriam capazes de vivenciar tal experiência, desenvolver capacidades cognitivas, físicas e emocionais, e aprender a LI. Tendo isso em mente, propusemos atividades que pensamos que ajudariam a atingir esses objetivos. Isto posto, nossa motivação em investigar este tema surgiu quando uma das atividades propostas não funcionou como esperávamos. A atividade era um jogo de tabuleiro no qual os alunos deveriam nomear a imagem em que o pino havia parado. No entanto, durante a atividade, percebemos que eles não conheciam aquele tipo de jogo e seu gênero, pois não sabiam, por exemplo, que deve-se seguir a ordem do tabuleiro, e que cada jogador tem sua vez. Identificamos que o problema não foi a língua em si (visto

\footnotetext{
${ }^{1}$ Optamos por usar o termo "língua estrangeira", pois este se refere ao uso/estudo de uma língua-alvo por falantes não-nativos em países onde tal linguagem geralmente não é um meio de comunicação local.
} 


\section{SEMINÁRIO DE PESQUISA EM CIÊNCIAS HUMANAS - SEPECH \\ Humanidades, Estado e desafios didático-científicos \\ Londrina, 27 a 29 de julho de 2016}

que eram capazes de nomear as imagens). Logo, tivemos de reconsiderar o tipo de conhecimento que não dominavam, e ensinar-lhes o que precisavam saber a fim de realizar e concluir a atividade com sucesso.

O objetivo principal deste trabalho foi o de investigar e compreender melhor se e como dominar o gênero textual "regra de jogo" é importante para a execução das atividades. Além disso, também buscamos examinar o que foi ensinado (além do próprio idioma) - e, consequentemente, o que as crianças podem aprender com o uso desse gênero textual.

\section{CRIANÇAS E O APRENDIZADO DE LI}

A introdução da LI na vida das crianças é bastante comum nos dias de hoje, e esse interesse tem crescido devido a inúmeros fatores, como a questão de que vivemos em um mundo cada vez mais globalizado (GRADDOL, 1997, 2006), fazendo com que estejamos em contato com línguas e culturas diferentes o tempo todo, e com que o conhecimento de outra língua seja fundamental. Além disso, também há o argumento de da quantidade total de tempo de exposição, fazendo com que as crianças apresentem atitudes mais positivas em relação à língua (CENOZ, 2003). Além disso, os pais têm levado em consideração fatores como o mercado de trabalho e qualidade de vida futura (TONELLI E VERIDIANO, 2011). Esses e outros fatores têm intensificado a tendência de iniciar o processo de aprendizagem da LI mais cedo, por isso muitos pesquisadores têm analisado esse tema (CAMERON, 2001; TONELLI 2005, 2008; CRISTOVÃO E TONELLI, 2010), e buscado formas de contribuir e reforçar o sucesso das crianças no que diz respeito à essa aprendizagem.

Algumas formas de ensinar as crianças são o uso de músicas (SIMIONATO e CHIMENTÃO, 2010), jogos/brincadeiras (BROUGÈRE, 2002; CHAGURI, 2009; CHAGURI E TONELLI, 2014), e histórias infantis (TONELLI, 2005). Neste trabalho, como já mencionado, nos concentramos no uso de jogos para o ensino de LI para crianças.

\section{O USO DE JOGOS NO ENSINO DE LI PARA CRIANÇAS}

O uso de jogos e atividades lúdicas para o ensino para crianças é uma tendência que é amplamente apoiada (BROUGÈRE, 2002; CHAGURI, 2009; CHAGURI E TONELLI, 2014), pois não só proporciona relaxamento, mas também incentiva o uso da língua de forma criativa e comunicativa. No entanto, não devem ser vistos como preenchimentos de tempo programados apenas para diversão, assim como apontado por Figueira (2010), os jogos precisam ser vistos não somente como uma atividade motivacional, mas também como uma atividade que ajuda na construção da linguagem.

De acordo com Yolageldili e Arikan (2011, p 220, tradução nossa), jogos envolvem "muitos fatores, tais como o uso de regras, o estímulo à cooperação, enquanto tornam a aprendizagem divertida" ${ }^{2}$. Além disso, motivam os alunos, pois fornecem contextos significativos e proporcionam competição; nos jogos, todos precisam

\footnotetext{
2 "many factors such as employing rules, fostering cooperation while making learning fun" (YOLAGELDILI and ARIKAN, 2011, p 220)
} 


\section{SEMINÁRIO DE PESQUISA EM CIÊNCIAS HUMANAS - SEPECH \\ Humanidades, Estado e desafios didático-científicos \\ Londrina, 27 a 29 de julho de 2016}

cooperar para chegar ao final do jogo, portanto os alunos acabam aprendendo a ideia de cooperação e interação social, e como Yolageldili e Arikan (2011, p 220, tradução nossa) dizem: "Acredita-se que quando a cooperação e interação reúnem-se com a diversão, a aprendizagem bem-sucedida torna-se mais possível". ${ }^{3}$ Cristovão e Gamero (2009, p 231) afirmam que, de acordo com Vygotsky (1993, p. 107), “(...) o jogo é a forma natural de trabalho da criança, a forma de atividade que lhe é inerente, a preparação para a vida futura", por isso os jogos são considerados indispensáveis na educação das crianças.

De acordo com Figueira (2010), as atividades lúdicas fornecem um ambiente, leve, favorável e agradável, tanto para a aprendizagem lexical quanto para a interação e é por isso que o aprendizado de outra língua tende a ser mais agradável quando acontece por meio delas. A ludicidade torna o conteúdo mais fácil aos olhos das crianças, portanto elas ficam mais interessadas na aula. Por isso, em nossa prática docente, decidimos colocar essas ideias em ação, pois concordamos com a ideia de que as crianças aprendem mais e melhor com a ajuda de jogos, considerando que a sala de aula se torna um ambiente mais leve e, como McCallum afirmou (1980 apud YOLAGELDILI e ARIKAN, 2011), usando jogos, o interesse e a motivação, que são alguns dos principais fatores para uma aprendizagem bem-sucedida, melhoram.

Brougère (2002) argumenta que é jogando que as crianças criam sua cultura lúdica ${ }^{4}$. Nas palavras de Brougère (2002, p 30), "O jogo é antes de tudo o lugar de construção (...) de uma cultura lúdica". Conforme exposto, o jogo cria um ciclo com a cultura; ele é, ao mesmo tempo, um produto cultural e uma criação cultural, “(...) o jogo produz a cultura que ele próprio requer para existir" (BROUGÈRE, 2002, p 30). Quando alguém joga um jogo, isso significa que as estruturas preexistentes e o como jogar foram aprendidos, ou seja, cultura foi adquirida; e também significa que a então aprendida cultura está sendo posta em prática - sendo assim criado um conjunto de estruturas e significados próprio do jogador.

\section{GÊNEROS TEXTUAIS}

Quando pensamos em gêneros textuais, pensamos neles como uma maneira de usar a língua; nas palavras de Hyland (2003b, p 18, tradução nossa), os gêneros são “(...) maneiras abstratas e socialmente reconhecidas de usar a linguagem para fins particulares"5 e para Millar (2011, p. 4), "Um gênero representa um grupo de textos que compartilham um propósito comunicativo. "6 Como Marcuschi (2002, p 35) aponta, gêneros textuais não são criações individuais, eles são "(...) formas socialmente aperfeiçoadas em práticas comunicativas." Cada gênero tem um propósito ou função, e como dito por Hyland (2003, p. 18, tradução nossa) "Nós não apenas escrevemos, nós

\footnotetext{
3 "It is believed that when cooperation and interaction are combined with fun, successful learning becomes more possible". (YOLAGELDILI and ARIKAN, 2011, p 220)

${ }^{4}$ Cultura lúdica é o conjunto de procedimentos que tornam os jogos possíveis, e que é necessária para que o jogador jogue.

5 “(...) abstract, socially recognized ways of using language for particular purposes" (HYLAND, 2003b, p $18)$

6 “A genre represents a group of texts that all share a communicative purpose.” (MILLAR, 2011, p. 4)
} 


\section{SEMINÁRIO DE PESQUISA EM CIÊNCIAS HUMANAS - SEPECH \\ Humanidades, Estado e desafios didático-científicos \\ Londrina, 27 a 29 de julho de 2016}

escrevemos algo para atingir algum propósito; é uma maneira de fazer com que as coisas sejam feitas".

A partir desta perspectiva, Figueiredo (2010, p 128, tradução nossa) acredita que, "Praticamente tudo o que fazemos envolve algum tipo de gênero" ligado a ideia de Bakhtin (1997 apud TONELLI, 2008) de que a comunicação verbal só é possível através da utilização de um gênero textual, e com a opinião de Marcuschi, que afirma que “ (...) é impossível se comunicar verbalmente a não ser por algum gênero, (...)" (2002, p 22)

Ter o conceito de gêneros textuais em mente e saber usar esses gêneros - mesmo sem se dar conta disso - é importante para os estudantes, pois fornece-lhes acesso a novas formas de comunicação que serão necessárias algum dia; eles melhoram a compreensão e a prática cultural/social, e provoca a consciência de gênero ${ }^{9}$ dos alunos. Figueiredo (2010) afirma que os gêneros nos permitem operar em sociedade e interagir socialmente, alcançando objetivos sociais, pois seus padrões não são aleatórios, eles foram estruturados de uma forma eficaz, e provaram-se funcionais.

O gênero "regras de jogo" é um gênero textual que tem o objetivo de fazer com que o jogador aja de uma determinada maneira. Ele fornece comandos que devem ser seguidos a fim de alcançar um resultado - o fim do jogo (MEDEIROS, 2009). Este gênero textual tem propósitos e usos reais e sociais no cotidiano das crianças, porque os jogos são parte de sua cultura e do mundo.

\section{METODOLOGIA}

Esta pesquisa é de natureza qualitativa, e baseia-se na metodologia de pesquisaação (SAGOR, 2000; LEWIN, 1948), forma de investigação baseada na autorreflexão, que procurar melhorar as práticas de ensino. Sagor (2000) a caracteriza como um processo realizado por e para aqueles que tomam a ação, além de apontar que uma das principais razões para se envolver neste tipo de pesquisa é ajudar o "ator" a melhorar suas próprias ações. Elliot (1997 apud BEHRENS e RODRIGUES, 2015, p 60) postula que esta metodologia "permite superar as lacunas existentes entre a pesquisa educativa e a prática docente, ou seja, entre a teoria e a prática, e os resultados ampliam as capacidades de compreensão dos professores e suas práticas, por isso favorecem amplamente as mudanças".

Esta pesquisa foi realizada no CEEI (Centro Estadual de Educação Infantil) da Universidade Estadual de Londrina (UEL) como parte do estágio obrigatório, que deve ser feito nos dois últimos anos do curso de Letras. O estágio no CEEI acontece em duplas - um aluno do terceiro ano e outro do quarto ano. O primeiro autor deste trabalho cumpriu o estágio obrigatório, e durante o segundo ano de estágio, Leonardo ${ }^{10}$ foi nosso parceiro.

Os alunos matriculados nesta escola são todos e exclusivamente filhos de funcionários da universidade, e os alunos que participaram desta pesquisa são da sala de

\footnotetext{
7 "We don't just write, we write something to achieve some purpose; it is a way to get things done". (HYLAND, 2003, p. 18)

8 "Virtually everything we do involves some kind of genre." (FIGUEIREDO, (2010, p 128)

${ }^{9}$ Genre awareness "the ability to select and use an appropriate genre based on a number of factors, including the purpose of communication, the context, and the people involved." (MILLAR, 2011, p 2)

${ }^{10}$ Nome fictício para preservação da identidade.
} 


\section{SEMINÁRIO DE PESQUISA EM CIÊNCIAS HUMANAS - SEPECH \\ Humanidades, Estado e desafios didático-científicos \\ Londrina, 27 a 29 de julho de 2016}

aula $E 5$, que é composta por crianças de quatro a cinco anos. Os alunos desta escola têm aulas em tempo integral, porém não têm aulas de LI com professores regulares, no entanto, estas são oferecidas a algumas salas desde 2013, como parte de um projeto ${ }^{11}$, e também como parte do estágio obrigatório estudantes Letras.

No contexto do ensino de LI para crianças como estágio obrigatório, um dos requisitos feito para os professores-alunos ${ }^{12}$ é o de escrever diários reflexivos, a fim de criar um processo reflexivo, um caminho em direção a transformação dos professores (LIBERALI, 1996). Para nossos dados, um diário de Leonardo e um diários do primeiro ano de estágio do primeiro autor foi selecionado. A escolha foi baseada no foco da pesquisa - o uso de jogos no contexto de ensino de LE para crianças.

\section{RESULTADOS}

\section{Diário do Primeiro Autor}

Tendo em vista que uma das abordagens de ensino de LI para crianças é o uso de jogos e brincadeiras (BROUGËRE, 2002; CHAGURI, 2009; CHAGURI E TONELLI, 2014), a ideia de usar um jogo de tabuleiro para revisar a matéria veio à nossa mente. Como mencionado anteriormente, acredita-se que o uso de jogos facilita a aprendizagem de línguas, especialmente para crianças, porque não só relaxa, mas também incentiva o uso da língua de forma criativa e comunicativa.

In the previous class, we did the board game activity with the students but it was not a completely success due to the fact that not all of them knew the how to play and the rules of a game board. In this class, the board game activity worked better because they were more familiar with the rules (one at a time, order of players, everyone throws the dice, order on the board,...) so they could play it more easily.

Excerto 1 - Excerto dos diários reflexivos

Ao analisar o diário reflexivo de 2014 do primeiro autor, é possível ver que o "erro" com o jogo estava no fato de que nós, professores, tínhamos em mente que os alunos já sabiam como jogar um jogo de tabuleiro, e o fato de não explicarmos as regras foi o que fez a atividade não ser tão produtiva quanto poderia ter sido - embora fosse uma atividade lúdica e, em teoria, seria bem-sucedida. No excerto 1, pode-se ver que as crianças não estavam familiarizadas com as regras básicas do jogo, e nós havíamos assumido que elas eram, o que as tornou incapazes de jogar como esperado. $\mathrm{Na}$ aula seguinte, nós ensinamos as regras, e eles puderam jogar melhor. Uma vez que "tudo o que fazemos envolve algum tipo de gênero"13 (FIGUEIREDO, 2010, p 128, tradução nossa), conhecer o gênero "regras de jogo" era essencial para o desenvolvimento da atividade, visto que esse fornecerá acesso a novas formas de comunicação que, provavelmente, serão necessárias algum dia (MILLAR, 2011).

\footnotetext{
11 “O Trabalho Do Professor No Ensino-Aprendizagem Da Língua Inglesa Para Crianças: Uma Proposta

De Formação Docente” coordenado pela Prof. Dr. Juliana Reichert Assunção Tonelli.

${ }^{12} \mathrm{O}$ termo professor-aluno é usado aqui para se referir aos alunos de graduação.

13 "everything we do involves some kind of genre" (FIGUEIREDO, 2010, p. 128)
} 


\section{SEMINÁRIO DE PESQUISA EM CIÊNCIAS HUMANAS - SEPECH \\ Humanidades, Estado e desafios didático-científicos \\ Londrina, 27 a 29 de julho de 2016}

The subject of the board game was a revision of the three main songs that were worked with on the previous semester (Twinkle Twinkle Little Star, Old Mac Donald Had a Farm and Head, shoulder, knees and toes).

Excerto2 - Excerto dos diários reflexivos

O conteúdo linguístico da atividade não parece ter sido o problema; o jogo era uma atividade de revisão que tinha o objetivo de fazer com que os alunos relembrassem o vocabulário relacionado às músicas trabalhadas no semestre anterior. De acordo com os diários, isso não apresentou grandes problemas; os alunos lembraram a maioria das palavras, e as relacionaram com as imagens. Às vezes, diziam palavras em Português (Excerto 3), mas os professores-alunos pediam para que um colega ajudasse a lembrar a palavra em inglês, o que tornou o processo de aprendizagem mais colaborativo e participativo. Desta forma, os alunos acabaram percebendo a ideia de cooperação e interação social, que, quando combinadas com diversão podem levar a uma aprendizagem mais bem-sucedida (YOLAGELDILI E ARIKAN, 2011).

Sometime the students would answer in Portuguese, so I would ask a colleague to help him or her, as a result, everybody could feel participatory, the learning process was collaborative and the knowledge was co-built. The student-student interactions had the goal of making them aware that they can help their friends and their friends can also help him or her, and that you can always use a little help from someone.

Excerto 3 - Excerto dos diários reflexivos

É mencionado no diário, que os alunos adquiriram cultura (Excertos 4 e 5); essa ideia está ligada (2002) a noção de Brougère de que jogando as crianças criam sua cultura lúdica. Jogos criam um ciclo com a cultura, e são, ao mesmo tempo, um produto cultural e uma criação cultural.

The contributions of this class for the students were diverse. First, they could remember everything they learned on the previous semester and see that they are really learning another language; second, they learned the rules, how a game board works and how to play it, and thus a pieck of culture was given to them. Third, they developed their ability of playing and working in groups, not only individually; and forth, they ended up, indirectly, learning some numbers when rolling the dice.

Excerto 4 - Excerto dos diários reflexivos 


\section{SEMINÁRIO DE PESQUISA EM CIÊNCIAS HUMANAS - SEPECH \\ Humanidades, Estado e desafios didático-cientificos \\ Londrina, 27 a 29 de julho de 2016}

This class contributed for the students' citizenship in the way that they were able to work together without major problems; they helped each other and respected their friends and their time. They also learned culture, the game board culture; something we thought they would already have when we first did it with them.

Excerto 5 - Excerto dos diários reflexivos

\section{Diários de Leonardo}

Os diários de Leonardo se concentraram mais em como as atividades foram aplicadas; e como como a aula aconteceu (passo a passo). No entanto, em alguns momentos, ele também descreveu algumas coisas que não aconteceram, em suas palavras, "de acordo com o plano" e dá o seu parecer e observações sobre.

Ao falar do jogo da memória utilizado em sala de aula, Leonardo não mencionou se o professor explicou as regras. No entanto, uma vez que éramos sua parceira de estágio e supervisora, e estávamos presentes, sabemos que as regras não foram explicadas explicitamente. No entanto, foi perguntado aos alunos se eles conheciam aquele jogo e suas regras, e eles disseram que já conheciam, e nós apenas acrescentamos informações que não foram mencionadas. Portanto, os estudantes jogaram com as regras em mente.

U segredo prıncıpal para começarem a ter maıs chances no jogo, serıa as crianças perceberem que os cartões de cores eram brancos, enquanto os cartões de números eram azuis. Porém, o desempenho dos alunos não foi bom na hora de encontrarem os cartões iguais. Eles conseguiram dizer os nomes das cores e dos números ao virarem-nos, que era o ponto principal da atividade, porém, não conseguiram desenvolver a parte do jogo, que era o que deveria lhes proporcionar diversão, talvez por falta de tempo. A atividade não mudou de rumo nem mesmo quando o aluno Afonso percebeu o fato que os cartões tinham cores diferentes (números azuis, cores brancos).

Excerto 6-Excerto dos diários reflexivos

No excerto 6, é possível ver que a opinião de Leonardo sobre o desempenho dos alunos não era positiva em termos do jogo, afirmando que os alunos não foram capazes de encontrar as cartas iguais, embora fossem capazes de dizer as palavras quando viravam as cartas. Nós acreditamos que o problema estava no fato de que eles estavam tendo dificuldades em tentar estabelecer uma conexão entre as imagens e posição na lousa. Visto que os alunos tinham idades entre quatro e cinco anos, pensamos que talvez pudessem haver muitos cartões para que eles fizessem a conexão e encontrassem os pares, ou seja, talvez o jogo estive muito difícil de resolver. 


\section{SEMINÁRIO DE PESQUISA EM CIÊNCIAS HUMANAS - SEPECH \\ Humanidades, Estado e desafios didático-científicos \\ Londrina, 27 a 29 de julho de 2016}

O aluno Gabriel teve dificuldades e não conseguiu achar o seu par, o que fez com que ele caísse em prantos e a professora Ana Beatriz tivesse que conversar com ele para contê-lo e explicar que nem sempre é possível vencer. Fora isso, todos os alunos se saíram bem, inclusive Lucas, a quem auxiliei para a execução da atividade. Por fim, todos os alunos conseguiram encontrar os pares de seus cartões e a atividade foi um sucesso.

Excerto 7 - Excerto dos diários reflexivos

O excerto 7 foi retirado do segundo diário reflexivo de Leonardo, no qual ele falou sobre a aula seguinte em as atividades começadas na aula anterior foram concluídas. Neste excerto, a ideia de Figueiredo (2010) sobre o fato de que os gêneros nos permitem operar em sociedade e interagir socialmente pode ser vista claramente, pois um aluno não foi capaz de encontrar um par correspondente e começou a chorar, logo, o professor-aluno tentou fazê-lo entender que ele não vai e não precisa ganhar tudo o tempo todo. Cristovão e Gamero (2009, p 231) afirmam que, de acordo com Vygotsky (1993) "(...) o jogo é a forma natural de trabalho da criança, a forma de atividade que lhe é inerente, a preparação para a vida futura" (VYGOTSKY, 1993 apud CRISTOVÃO e GAMERO, 2009, p 231). Além disso, quando crianças participam em jogos com regras, eles estão sendo preparados para a vida adulta, ou seja, estão aprendendo a viver uns com outros em sociedade (SOLER, 2005). Esta é a razão pela qual jogar/brincar é indispensável na educação das crianças; tal ato permite que elas interajam com os outros, com o mundo, com a realidade, e que elas façam parte da sociedade.

\section{OBSERVAÇÕES FINAIS}

Para usar jogos em sala de aula, é extremamente importante que o professor os planeje cuidadosamente antes de aplica-los, e que as crianças tenham as regras bem compreendidas em mente antes de jogar. Quando o aluno não compreende as regras, ele pode perder o interesse no jogo ou atividade, portanto, estas devem ser claras e sem muita complexidade a fim de motivar o aluno, fazendo com que ele se interesse pelo desafio e pela vontade de vencer.

Resumindo os resultados deste trabalho, parece seguro concluir que o gênero "regras de jogo" é totalmente significativo para a aprendizagem dos alunos, pois permite-lhes uma familiarização com um gênero (mesmo que inconscientemente). Em outras palavras, as crianças vão conhecer o gênero sem saber disso. Além disso, os alunos são expostos a este novo gênero textual, o que faz com que este se torne mais presente na vida escolar dos alunos, o que pode evitar futuras dificuldades com outros novos gêneros.

Ao estabelecer uma compreensão dos dados coletados, é possível ver que conhecer as regras do jogo faz com que os estudantes estejam dispostos a jogá-lo, e mais propensos a atingir os objetivos principais das atividades. Da mesma forma, as crianças aprendem habilidades essenciais para a vida em sociedade, que requer lidar com regras a todos os momentos, assim como nos jogos de regras, que desenvolvem 


\section{SEMINÁRIO DE PESQUISA EM CIÊNCIAS HUMANAS - SEPECH \\ Humanidades, Estado e desafios didático-científicos \\ Londrina, 27 a 29 de julho de 2016}

questões importantes, tais como a adequação a limites, a criação de estratégias, a cooperação e competição. Além disso, os alunos podem perceber a aprendizagem significativa e cooperativa, e a participação do "outro", enquanto aprende a LI.

Com esta pesquisa, podemos ver melhor que os professores de LI para crianças ensinam não só a língua - vocabulário, gramática, ortografia ... Um exemplo do que foi ensinado com o uso desse gênero é a cultura; as crianças puderam aprender (ou aprender mais) sobre o jogo e foram capazes de co-construir sua cultura lúdica e, ao mesmo tempo, a "cultura do adulto" também. (BROUGÈRE, 2002).

O presente estudo revelou que gerar dados a partir de seus próprios trabalhos promove o crescimento e desenvolvimento contínuo, construindo, portanto, um profissional reflexivo, que vê cada lição “(...) como uma investigação empírica sobre os fatores que afetam o ensino e a aprendizagem"14 (SAGOR, 2000, p 7, tradução nossa). Finalmente, é importante dizer que concordamos com a ideia de que refletir sobre os resultados de cada trabalho pode dar ao professor oportunidades de pensar em sua própria prática, e em como ela poderia ser melhorada, desenvolvendo assim um “(...) maior domínio da arte e ciência de ensinar" ${ }^{\prime 15}$ como afirma Sagor (2000, p 7, tradução nossa).

\section{REFERENCES}

BEHRENS, Marilda Aparecida; RODRIGUES, Daniela Gureski. Paradigma emergente: um novo desafio. IN: Pedagogia em Ação, v. 6, n. 1, mar. 2015. ISSN 2175-7003.

BROUGÈRE, Gilles. A criança e a cultura lúdica. In: KISHIMOTO, Tizuko Morchida (Org): O brincar e suas teorias. São Paulo: Pioneira Thomson Learning, 2002. p. 19-32.

CAMERON, L. Teaching languages to young learners. Cambridge: CUP, 2001.

CENOZ, Jasone. The influence of age on the acquisition of English: General Proficiency, Attitudes and Code Mixing. IN: MAYO, M. De P. G.; LECUMBERRI, M. L. G. Age and Acquisition of English as a Foreign Language, 2003. Ps: 77-80.

CHAGURI, J. O ensino de espanhol com atividades lúdicas para aprendizes brasileiros. Revista X, v. 2, 2009.

CHAGURI, J. de P.; TONELLI, J. R. A. O jogo na sala de aula estrangeira para crianças. Folio - Revista de Letras, v. 6, p. 167-187, 2014.

CRISTOVÃO, V. L. L.; TONELLI, J.R.A. O papel dos cursos de Letras na formação de professores de inglês para crianças. Calidoscópio, v.8, n.1, p. 65- 76, 2010.

; GAMERO, Raquel. BRINCAR APRENDENDO OU APRENDER BRINCANDO? O INGLÊS NA INFÂNCIA. IN: Trab. Ling. Aplic., Campinas, 48(2): 229-245, Jul./Dez. 2009.

\footnotetext{
14 “(...) as an empirical investigation into factors affecting teaching and learning” (SAGOR, 2000, p 7).

15 "(...) greater mastery of the art and science of teaching”
} 


\section{SEMINÁRIO DE PESQUISA EM CIÊNCIAS HUMANAS - SEPECH \\ Humanidades, Estado e desafios didático-científicos \\ Londrina, 27 a 29 de julho de 2016}

FIGUEIRA, C. D. de S. O Envolvimento de Crianças na Aula de Língua Estrangeira. IN: ROCHA, C. H.; TONELLI, J. R. A; SILVA, K. A. da. Língua Estrangeira para crianças: Ensino-aprendizagem e Formação Docente. Campinas, SP: Pontes Editores, 2010 .

FIGUEIREDO, D. Context, register and genre: implications for language education. IN: Revista Signos, 43 / Número Especial Monográfico No 1, p. 119-141, 2010.

GRADDOL, David. English next. London: British Council, 2006.

. The future of English. London: British Council, 1997.

HYLAND, Ken. Genre-based pedagogies: A social response to process. IN: Journal of Second Language Writing 12, ps 17-29. 2003.

HYLAND, Ken. Second Language Writing. New York: Cambridge University Press. 2003 b.

LEWIN, Kurt. Action Research and Minority Problems. 1948. P. 34-46.

LIBERALLI, F. C. O Desenvolvimento Reflexivo do Professor. The ESP, São Paulo, v. 17, n. 1, p. 19-37, 1996.

MARCUSCHI, L. A. Gêneros textuais: definição e funcionalidade. In: DIONISIO, A. P. et al. (org.) Gêneros textuais \& ensino. Rio de Janeiro: Lucerna, p 19 - 36. 2002.

MEDEIROS, Neilson Alves de. A Construção da textualidade no gênero 'regra de jogo' em produções orais de crianças da pré-escola. 104 ps. Master thesis (Master in Linguistic) - Paraíba Federal University, João Pessoa, 2009.

MILLAR, Diane. Promoting Genre Awareness in the EFL Classroom. IN: English Teaching Forum, number 2. 2011.

SAGOR, Richard. What is action research? IN: . Guiding school improvement with action research. ASCD. 2000.

SIMIONATO, C. R.; CHIMENTÃO, L. K. A MÚSICA: um gênero a ser explorado no ensino de língua inglesa. IN: O Professor PDE e os desafios da escola pública paranaense. V. 1. 2010.

SOLER, R. Brincando e aprendendo com os jogos cooperativos. Rio de Janeiro: Sprint, 2005.

TONELLI, . 2005. Histórias infantis no ensino da língua inglesa para crianças. Londrina, PR. Masters thesis. Universidade Estadual de Londrina, 270 p. 


\section{SEMINÁRIO DE PESQUISA EM CIÊNCIAS HUMANAS - SEPECH \\ Humanidades, Estado e desafios didático-científicos \\ Londrina, 27 a 29 de julho de 2016}

TONELLI, . O uso de histórias infantis no ensino de inglês para crianças: analisando o gênero textual história infantil sob a perspectiva do interacionismo sóciointeracionismo sócio-discursivo discursivo discursivo. IN: Acta Scientiarum Language and Culture. Maringá, v. 30, n. 1, p. 19-27, 2008.

TONELLI, Juliana Reichert Assunção. Histórias infantis e ensino de Inglês para crianças: reflexões e contribuições. IN: Revista de C. Humanas, Viçosa, v. 13, n. 2, p. 297-315, jul./dez. 2013.

; VERIDIANO, P. A influência das crenças no ensino aprendizagem de língua inglesa para crianças: o envolvimento da comunidade escolar. IN: SILVA, K. A. da. (Org.) Crenças, discursos e linguagem. v.2. Campinas, SP: Pontes Editores, 2011.

YOLAGELDİII, Gülin; ARIKAN, Arda. Effectiveness of Using Games in Teaching Grammar to Young Learners. IN: Elementary Education Online, 10(1), 219-229, 2011. 